BMJ Open

Diabetes

Research

\& Care

\title{
Association between metformin and neurodegenerative diseases of observational studies: systematic review and meta-analysis
}

\author{
Fan Ping, Ning Jiang, Yuxiu Li (D)
}

To cite: Ping F, Jiang N, Li Y. Association between metformin and neurodegenerative diseases of observational studies: systematic review and meta-analysis. BMJ Open Diab Res Care 2020;8:e001370. doi:10.1136/ bmjdrc-2020-001370

- Additional material is published online only. To view, please visit the journal online (http://dx.doi.org/10.1136/ bmjdrc-2020-001370).

Received 1 April 2020 Revised 16 June 2020 Accepted 27 June 2020
Check for updates

(C) Author(s) (or their employer(s)) 2020. Re-use permitted under CC BY-NC. No commercial re-use. See rights and permissions. Published by BMJ.

Department of Endocrinology, Peking Union Medical College Hospital, Beijing, China

Correspondence to Dr Yuxiu Li;

liyuxiu@medmail.com.cn

\section{ABSTRACT}

Background and aims Aging becomes a growing global concern with an increased risk of neurodegenerative diseases (NDs) that mainly consist of cognitive decline and Parkinson disease (PD). As the most commonly prescribed antidiabetic drug, metformin has been shown to have inconsistent roles in the incidence of NDs. We performed a systematic review and meta-analysis of observational studies to evaluate the effect of metformin exposure on onset of NDs.

Methods The observational studies that investigated the associations between metformin and the incidence of NDs were searched in MEDLINE, Embase and Cochrane Library databases. A random-effect model was performed using STATA to calculate the combined ORs.

Results In total, 23 comparisons out of 19 studies with 285966 participants were included. Meta-analysis found there was no significant effect on incidence of all the subtypes of NDs with metformin exposure (OR 1.04, $95 \% \mathrm{Cl} 0.92$ to 1.17). However, metformin monotherapy was associated with a significantly increased risk of PD incidence compared with non-metformin users or glitazone users (OR $1.66,95 \% \mathrm{Cl} 1.14$ to 2.42 ).

Conclusion Metformin has failed to demonstrate a beneficial effect on NDs. In addition, it may increase the risk of PD development. In light of current results, how metformin would impact NDs, especially the potential risk of PD, needs to be scrutinized. The underlying mechanisms are vital to achieve some more profound understanding on the regimen.

Trial registration number CRD 42019133285.

\section{INTRODUCTION}

Aging becomes a global growing issue, accompanied by an increase in neurodegenerative diseases (NDs) related to aging. ND is an umbrella term for various types of central nervous system (CNS) degenerative disorders, including dementia, Alzheimer disease (AD), Parkinson disease (PD) and mild cognitive impairment. Unfortunately, these disorders caused by progressive degeneration or/ and death of neurons are currently without a disease-specific cure and lead to a heavy social and financial burden. The clinical trials of the new drugs for NDs are currently at a stagnant

\section{Significance of this study}

What is already known about this subject?

- Neurodegenerative diseases (NDs) caused by progressive degeneration or/and death of neurons are associated with insulin action in central nervous system.

- As a first-line antidiabetic pharmacotherapy, whether metformin is protective or detrimental to NDs remains controversial.

What are the new findings?

- Metformin was found to play a neutral role on the incidence of the overall NDs.

- Subgroup analysis showed metformin exposure increased the risk of Parkinson disease (PD) by $66 \%$ (OR $1.66,95 \% \mathrm{Cl} 1.14$ to 2.42 ).

How might these results change the focus of research or clinical practice?

- These results indicate that the risk-benefit balance of metformin should be carefully evaluated before being prescribed as the first-line therapy for patients with diabetes mellitus with PD or deemed at high risk of PD.

- Further studies are required to render a better understanding of the underlying pathogenic mechanism.

stage with a frustrating failure to success ratio of 100:1. ${ }^{1}$ Dementia is the most common degenerative disease of the nervous system, with an estimate of 40-50 million people affected worldwide. ${ }^{2}$ It is characterized by a progressive decline of cognitive function. As the second most common type of NDs, the global number of people living with PD more than doubled from 2.5 million in 1990 to 6.1 million in 2016, which is characterized with movement impairment. ${ }^{3}$ However, the increase was not solely due to aging because the age-standardised prevalence rates were also increased. More and more studies have shown that both dementia and PD are related to the pathogenesis of insulin resistance, which has been regarded as the 


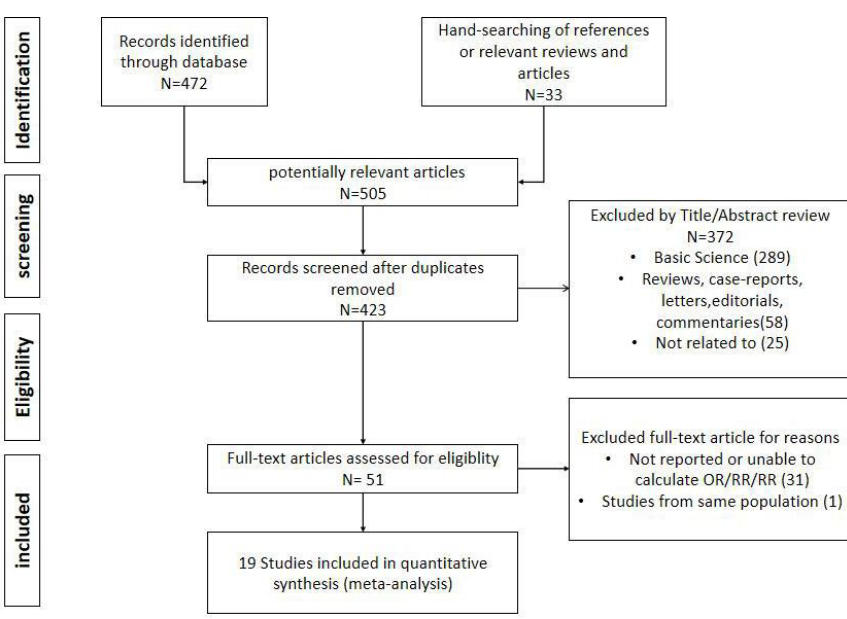

Figure 1 Flow diagram summarizing study identification and selection. $\mathrm{RR}$, relative risk.

most modifiable risk factor. Growing evidence suggests that the mesolimbic dopamine system is finely tuned by insulin action in the brain. Dopamine might mediate the central and peripheral insulin sensitivities, which indicate the relationship between NDs and glucose metabolism. ${ }^{4}$ Furthermore, some kinds of diabetes drugs have been reported to have important impacts on the pathogenesis of these two major kinds of NDs.

As a top-tier drug for the treatment of type 2 diabetes mellitus (T2DM), the role of metformin in dementia and PD is highly inconsistent, both in animal models and in clinical studies. At present, randomized controlled trial (RCT) studies on metformin in the clinical study of the degenerative nervous system were very few, which may be limited for the nature of NDs, which takes a long time to develop. It is insufficient to evaluate the risk changes of NDs in such a relatively short term. To better understand this issue, we performed a systemic review with metaanalysis of available clinical observational studies that investigate the effect of metformin on ND development.

\section{SUBJECTS AND METHODS}

A completed Strengthening the Reporting of Observational Studies in Epidemiology statement-checklist is presented in online supplementary table S1.

\section{Search strategy}

This systematic review was performed according to a prespecific protocol registered at PROSPERO and was reported in line with the Preferred Reporting Items for Systematic Reviews and Meta-Analyses guidelines. A literature search of MEDLINE, Embase and Cochrane Library database through January 2020 was conducted by two study investigators independently for all relevant articles on the incidence of NDs with metformin exposure. The key terms, including 'metformin', 'neurodegenerative Diseases', 'Parkinson Disease', 'Alzheimer Disease', 'Dementia', and 'Cognitive disorder' were searched in the article titles, abstracts and keywords. We also scrutinized the reference lists of the identified reviews, reports and other related articles to find additional pertinent studies. Language was limited to English.

\section{Selection criteria and quality assessment}

Published studies will be included if they fulfilled the following criteria: (1) evaluated and clearly defined exposure to metformin; (2) the primary outcome was incidence of various NDs; (3) the study reported either relative risks (RRs), HRs, and ORs or provided data for their calculation; and (4) the eligibility conditions for participants were given, or matching criteria were described for matched studies. The studies of basic science, reviews, case reports, letters, editorials, commentaries or articles not written in English will be excluded. The flow diagram summarizing study identification and selections is shown in figure 1 .

The methodological quality of case-control and cohort studies was assessed by two authors independently using the 9-star Newcastle-Ottawa Scale (NOS), while cross-sectional studies were evaluated by the Agency for Healthcare Research and Quality (AHRQ) methodology checklist with 11 items. Any discrepancies were resolved by a joint re-evaluation of the original article. We consider the studies with a cumulative NOS score of $\geq 7$ or a AHRQ score of $\geq 8$ as high quality. ${ }^{56}$ Then, sensitivity and subgroup analyses were carried out on the basis of quality assessment.

\section{Data abstraction and statistical analysis}

Data were independently abstracted onto a standardized form by two reviewers. The following data were collected from each study: study design, location, time period of study/year of publication, country of the population studied, primary outcome reported, total number of persons in each group, ORs, and 95\% CIs with or without adjustment for confounding factors. Studies comparing participants treated with metformin or referring to participants with diabetes mellitus (DM) but not treated with metformin or subjects without DM were adopted if the status of therapy or reference groups were clearly classified in the study.

All analyses were performed using STATA V.14.2. Because the incidence of outcome was relatively low, ORs were considered as approximation of RRs. Adjusted ORs reported in studies were used for analysis, so as to account for confounding variables. Combined ORs with corresponding $95 \%$ CI were used to assess the association between metformin and incidences of NDs. Natural logarithm of OR (LnORs) were considered to obey normal distribution. LnORs and the corresponding in lower limits and $\ln$ upper limits were used as data points in pooling analysis.

Heterogeneity among studies was assessed using $I^{2}$ statistic, for which statistically moderate heterogeneity was found $\left(I^{2}>0.5\right)$; the random-effects model was used to combine results. Publication bias was investigated by Begg's test and funnel plot analysis. Prespecified 
subgroup analysis was performed to investigate the source of heterogeneity by several major covariates based on the subtypes of NDs, race and study design. A metaregression was performed to explore possible explanations for between-subgroup heterogeneity.

\section{RESULTS}

Search results

Of the 505 potentially relevant studies identified using our search criteria, 23 comparisons out of 19 studies comprising a total of 285966 participants fulfilled our inclusion criteria and were involved in the meta-analysis. A total of 13 population-based cohorts, 3 cross-sectional studies and 3 case-control studies from 19 reports (4 of them ${ }^{7-9}$ provided more than one independent cohort for different disease types or age groups) explored the risk of cognitive disorder, including cognitive dysfunction, dementia or $\mathrm{AD}$ among users of metformin, with one of the studies published only in conference abstract form. ${ }^{10}$ There are three population-based cohort studies exploring the association of PD and metformin exposure. Among them, the control group in one study included the patients with DM treated with glitazone, ${ }^{8}$ while in the other two studies, the control groups were just patients with DM without metformin treatment. ${ }^{711}$

\section{Characteristics of the included studies}

The characteristics of these studies are shown in table 1 .

Among a total of 20 comparisons out of 17 observational studies focusing on cognitive impairment (including cognitive dysfunction, dementia and $\mathrm{AD}$ ), 5 cohorts from Taiwan used the same database of the National Health Insurance (NHI), but the findings were conflicting. Hsu et al reported in 2011 that, compared with T2DM without any medication, metformin alone decreased the risk of dementia by $24 \%$ (HR $0.76,95 \%$ CI 0.58 to 0.98$).{ }^{12}$ The second study in 2014 by Cheng et al found that metformin treatment alone in T2DM failed in reducing dementia compared with sulfonylureas (SUs) (HR 0.82, 95\% CI 0.52 to 1.28 ). ${ }^{13}$ Another study of the same year initiated by Huang et al also failed to confirm the benefits of metformin in $\mathrm{AD}$ incidence as both the monotherapy (HR $0.69(0.28-1.71)$ or the combination therapy (HR $0.57,95 \%$ CI 0.26 to 1.26 ).$^{14}$ However, it is unsettling that the cohort study published in 2017 by Kuan et al observed that the metformin exposure increased all-cause dementia by $66 \%$ (HR 1.66, 95\% CI 1.35 to 2.04), including both AD (HR 2.13, 95\% CI 1.2 to 3.79 ) and vascular dementia (HR 2.3, $95 \%$ CI 1.25 to 4.22). ${ }^{7}$ However, the latest study in 2019 by Tseng found the incidence of dementia in metformin users decreased by $29 \%$ (HR $0.71,95 \%$ CI 0.63 to 0.79 ) compared with metformin never users in newly diagnosed diabetes. ${ }^{15}$ Results of cohort studies from other countries or regions were mostly neutral, ${ }^{916-18}$ but there were also improvements ${ }^{1922}$ or exacerbations ${ }^{910}$ on cognitive decline with metformin. Among them, a cohort study of US veterans found metformin, compared with SU, had a neutral effect in patients with DM older than 75 years and a protective effect on individuals between 65 and 75 years. ${ }^{18}$ By contrast, a population-based study in UK demonstrated that HR of incident dementia with metformin users increased by $42 \%$ (95\% CI $1.02 \%$ to $1.98 \%, \mathrm{p}=0.038$ ), and incident $\mathrm{AD}$ also had an increasing trend but failed to reach the statistical significance (HR $1.6(0.87-2.93)$, $\mathrm{p}=0.131){ }^{9}$ There were three cross-sectional studies: the first study of Japan concluded a protective effect of metformin in cognitive dysfunction (OR 0.59, 95\% CI 0.35 to 0.99$).{ }^{19}$ The second study from the USA found that patients with diabetes with metformin intake were less likely to have cognitive decline effects (OR 0.52, 95\% CI 0.24 to $1.0 ; \mathrm{p}=0.05) .{ }^{20}$ The latest one from Northern Ireland showed a trend toward higher cognitive dysfunction in hyperglycemia with metformin treatment (OR $1.29,95 \%$ CI 0.96 to 1.74$).{ }^{21}$ In all three case-control studies published so far, two studies from $\mathrm{UK}^{22}$ and Australia, ${ }^{23}$ respectively, had concluded that cognitive performance became worse (OR 1.73, 95\% CI 1.11 to 2.68, and OR $2.23,95 \%$ CI 1.05 to 4.75 ) in the metformin intake group; the latter one also found the association was weakened after adjusting for vitamin $\mathrm{B}_{12}$ levels (OR $1.75,95 \% \mathrm{CI} 0.81$ to 3.78 ). Another German case-control study yielded a neutral result (with vs without metformin OR $0.96,95 \%$ CI 0.88 to 1.04$){ }^{24}$

To date, there have been three cohort studies evaluating metformin effect on PD incidence, two of which were from the NHI database in Taiwan. Kuan et al found metformin exposure increased PD incidence significantly compared with non-metformin cohort (HR 2.27 $(1.68-3.07)) .^{7}$ However, the study by Wahlqvist et al in 2012 did not reach a similar conclusion with metformin exposure on PD (HR 1.3, 95\% CI 0.69 to 2.46). ${ }^{11}$ Another study performed in Norway concluded that metformin use was associated with a significantly higher incidence of PD compared with glitazone use (HR 1.39, 95\% CI 1.06 to 1.85$)$.

\section{Quality of included studies}

Online supplementary table S2-S4 described the methodological quality of each included study. Most studies were adjusted for age, gender, education, body mass index, comorbidity, DM complications and other medications, whereas a few studies were adjusted for glycated hemoglobin $(\mathrm{HbA1c})$ value $(4 / 19)$ and vitamin $\mathrm{B}_{12}$ level $(2 / 19)$ (online supplementary table S5 shows the variables adjusted for in each study). The median NOS score for all of the cohort studies and case-control studies (full score of 9) was 7, which means they can be considered as high quality except for two studies with a relatively small sample size. ${ }^{1016}$ The other limitations of these two studies are (1) metformin exposure and adherence to treatment were determined by self-reports; (2) subjects with missing values on covariates were omitted ${ }^{16}$; and (3) the follow-up time was not long enough for outcomes to occur. ${ }^{10}$ The median AHRQ score for three cross-sectional studies 


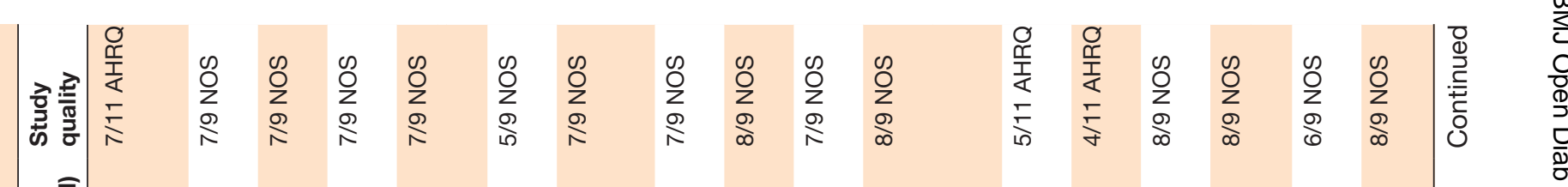

כ) -

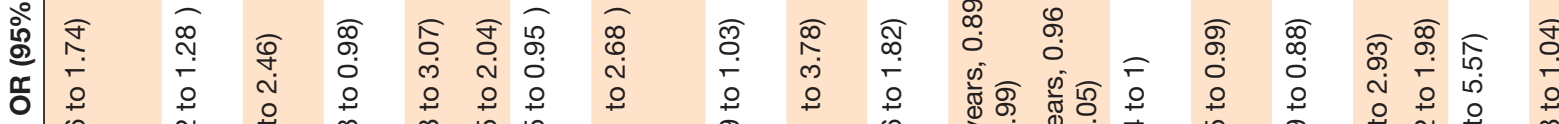

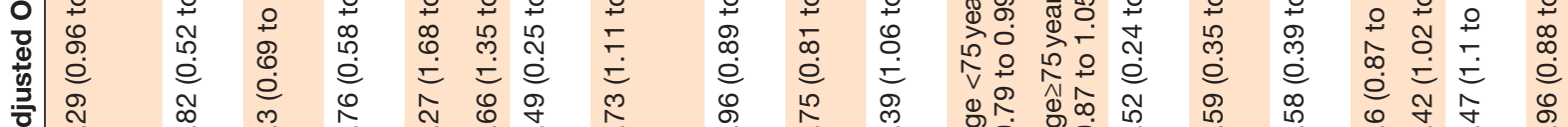

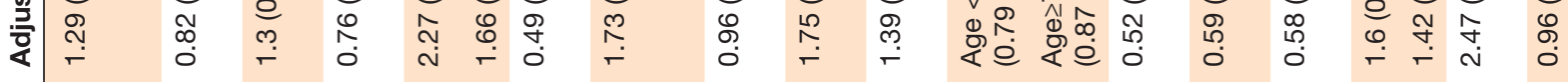

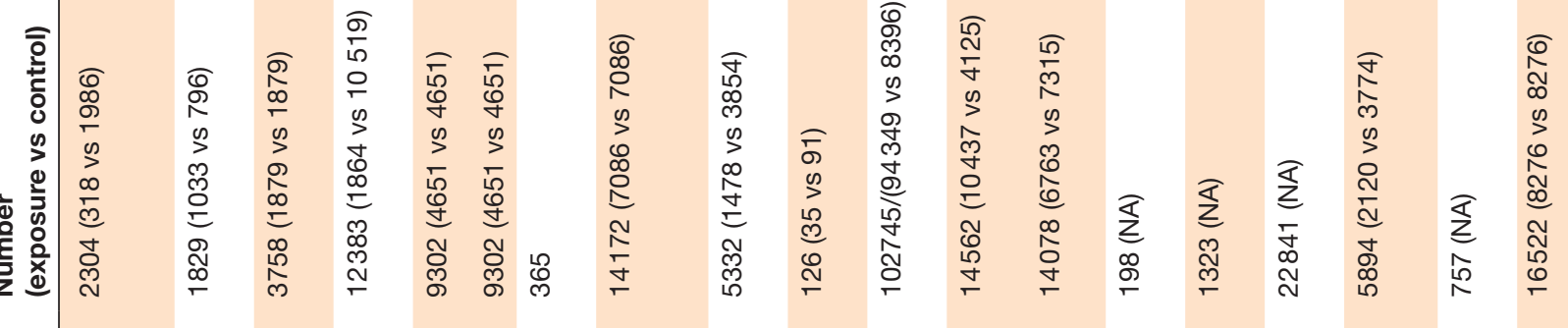

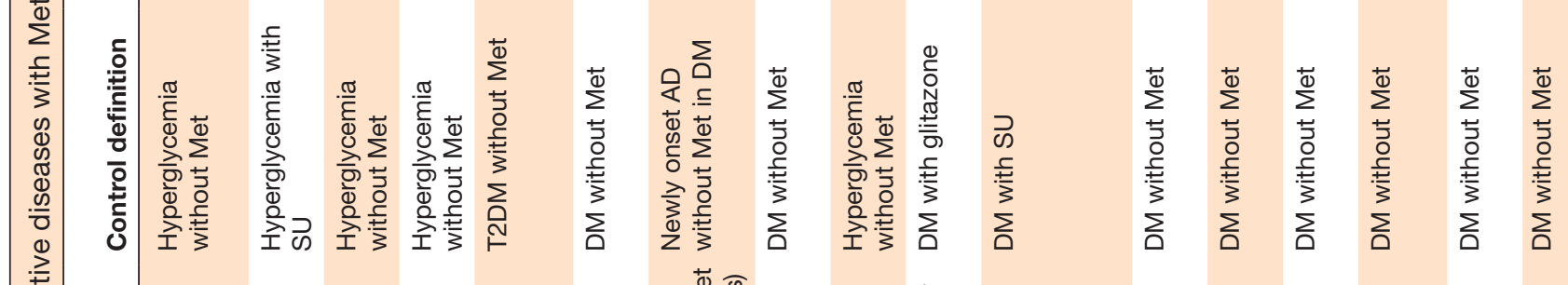

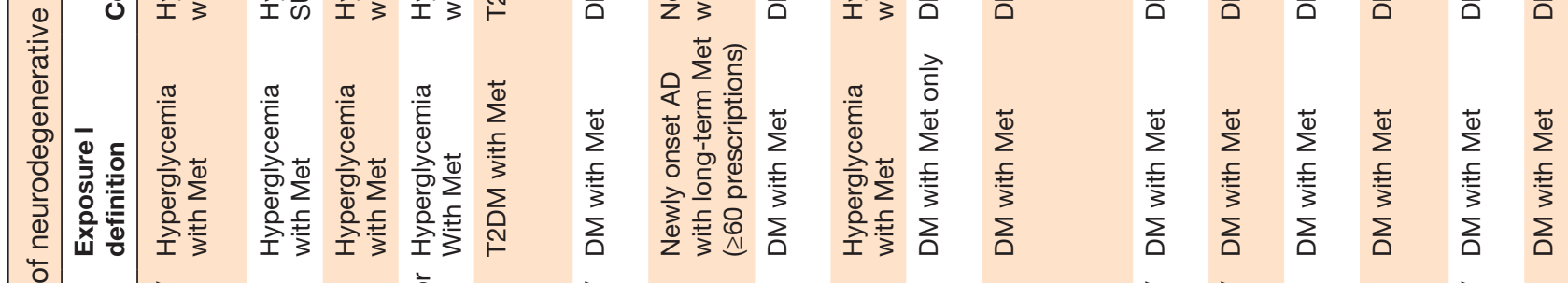

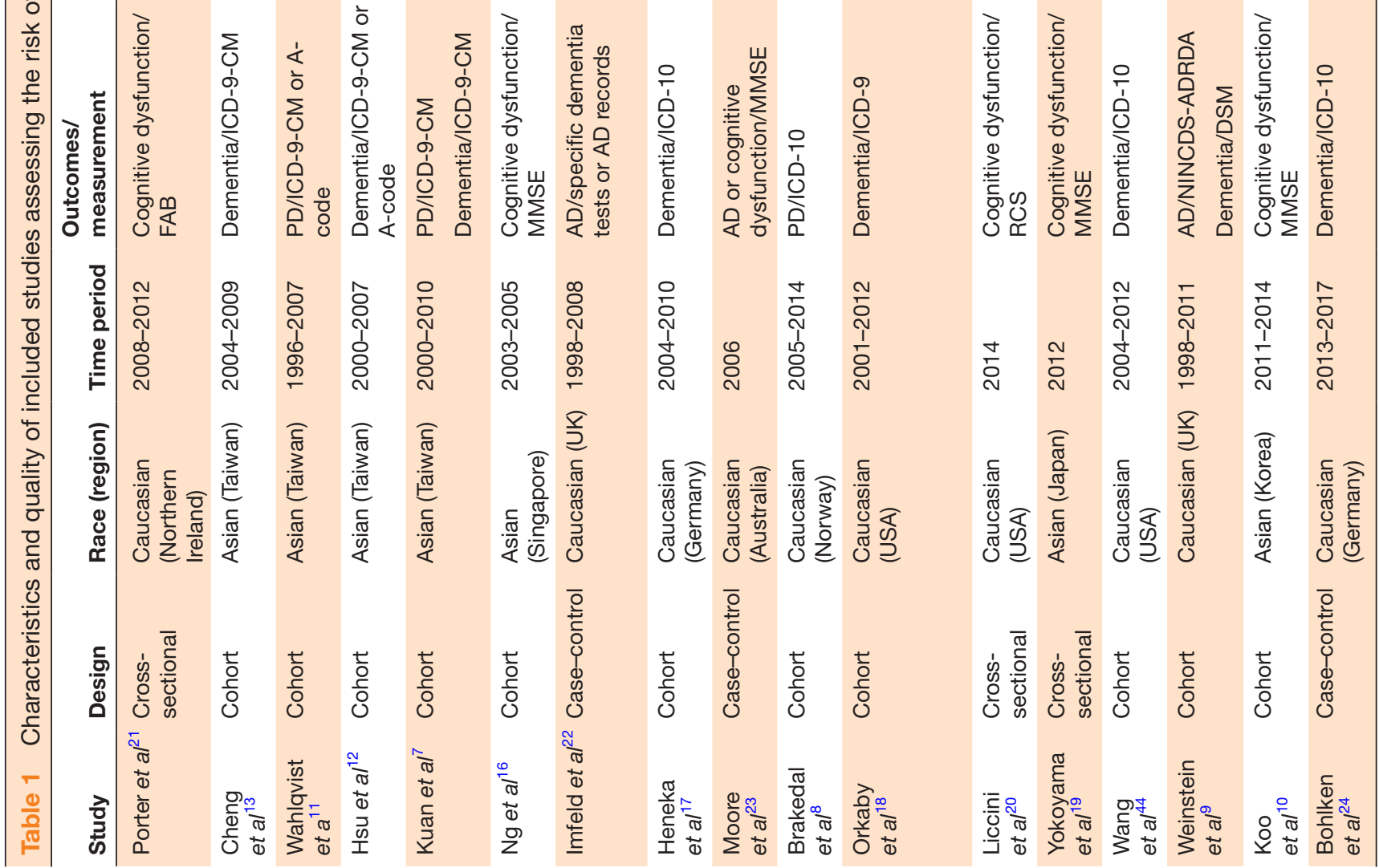




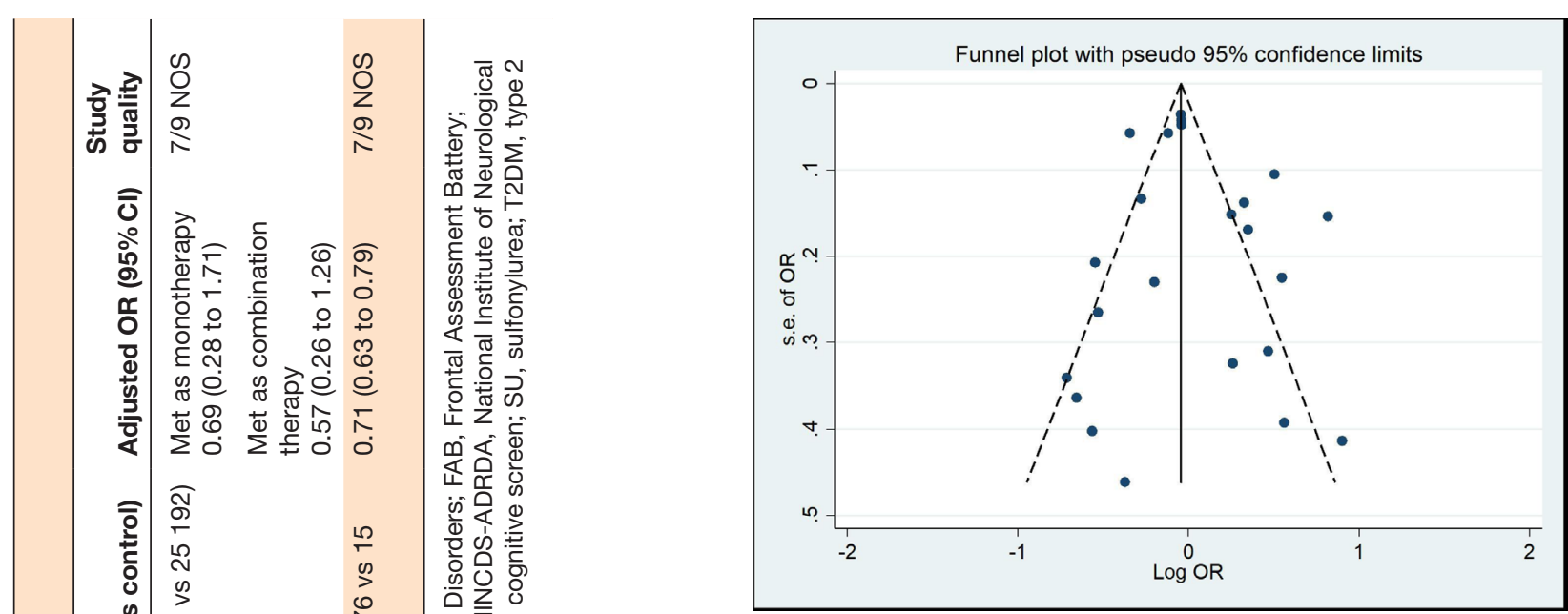

Figure 2 Funnel plot of log OR for neurodegenerative disease incidence with metformin exposure.

was 5 (range 4-7, full score of 11), which was somewhat self-explanatory on the overall quality of these three studies. ${ }^{19-21}$

\section{Risk of NDs and publication bias}

On meta-analysis of all the included studies, the application of metformin has a neutral effect on the onset of general NDs (OR 1.04, 95\% CI 0.92 to 1.17 ). However, the results showed considerable heterogeneity $\left(\mathrm{I}^{2}=84.6 \%\right.$, Cochran's $Q$ test $p=0.00)$. The funnel plot showed there was no significant asymmetry (figure 2). Also, Begg's test did not reveal the risk of publication bias $(\mathrm{Z}=0.32$, $\mathrm{p}=0.751>0.05)$.

\section{Subgroup analysis}

We performed preplanned stratified analyses of studies based on the category of disorders, race and study design (online supplementary table S6).

\section{Quality of observational studies}

Only five studies were considered as low quality with a relatively small sample size in total $(n=4947)$. The lowquality studies yielded an OR of 0.86 (95\% CI 0.5 to 1.48 ) compared with high-quality studies $(\mathrm{n}=29436$, OR 1.07 , $95 \%$ CI 0.94 to 1.21 ) (figure 3 ).

\section{Subtypes of ND}

The pooled adjusted OR for PD was 1.66 (95\% CI 1.14 to 2.42); that for $\mathrm{AD}$ was 0.9 (95\% CI 0.47 to 1.73 ); and that for dementia was 0.96 (95\% CI 0.85 to 1.09) (figure 4).

Race

Considering the influence of racial differences, the pooled OR of 11 Asian studies was 0.97 (95\% CI 0.69 to 1.36), whereas combined OR for 12 Caucasian comparisons was 1.04 (95\% CI 0.93 to 1.15). (online supplementary figure $\mathrm{S} 2$ )

\section{Study design}

Regarding the study design, the pooled OR for 17 cohort studies (including prospective and retrospective) was 


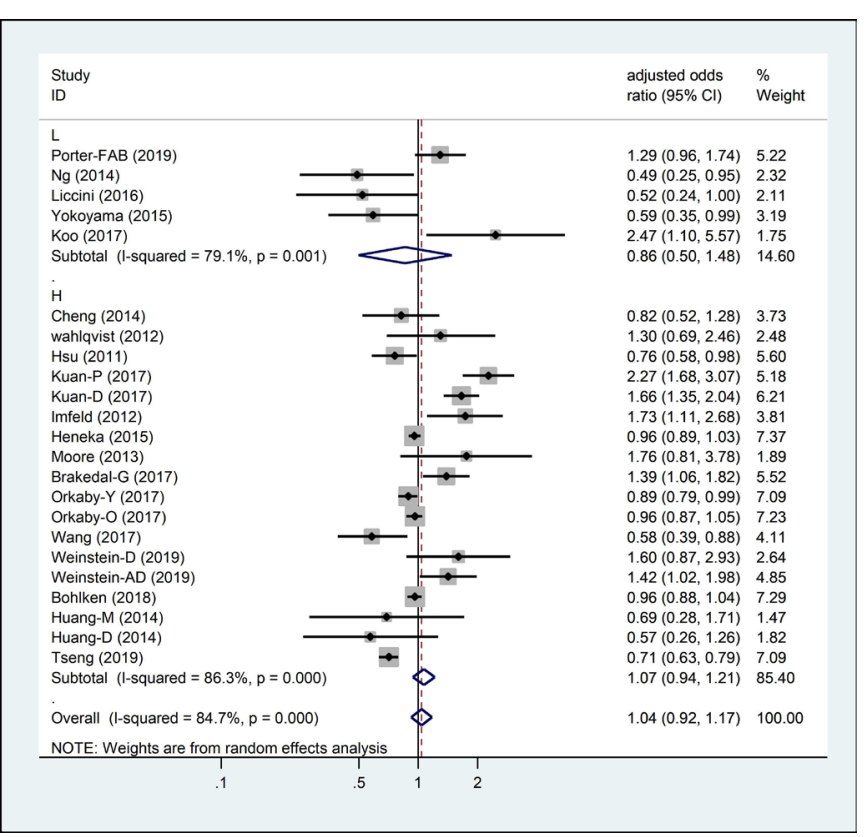

Figure 3 Adjusted OR for metformin exposure on neurodegenerative disease incidence subgrouped by research quality stratification: L, low quality; $\mathrm{H}$, high quality.

1.04 (95\% CI 0.89 to 1.21$), 0.77$ (95\% CI 0.41 to 1.46 ) for three cross-sectional studies and 1.33 (95\% CI 0.82 to 2.16) for three case-control studies, respectively (online supplementary figure S3).

\section{Sensitivity analysis}

To evaluate the influence of individual study in overall estimate, we performed a sensitivity analysis by omitting each study in turn. As shown in online supplementary figure S1 with each of the studies individually removed,

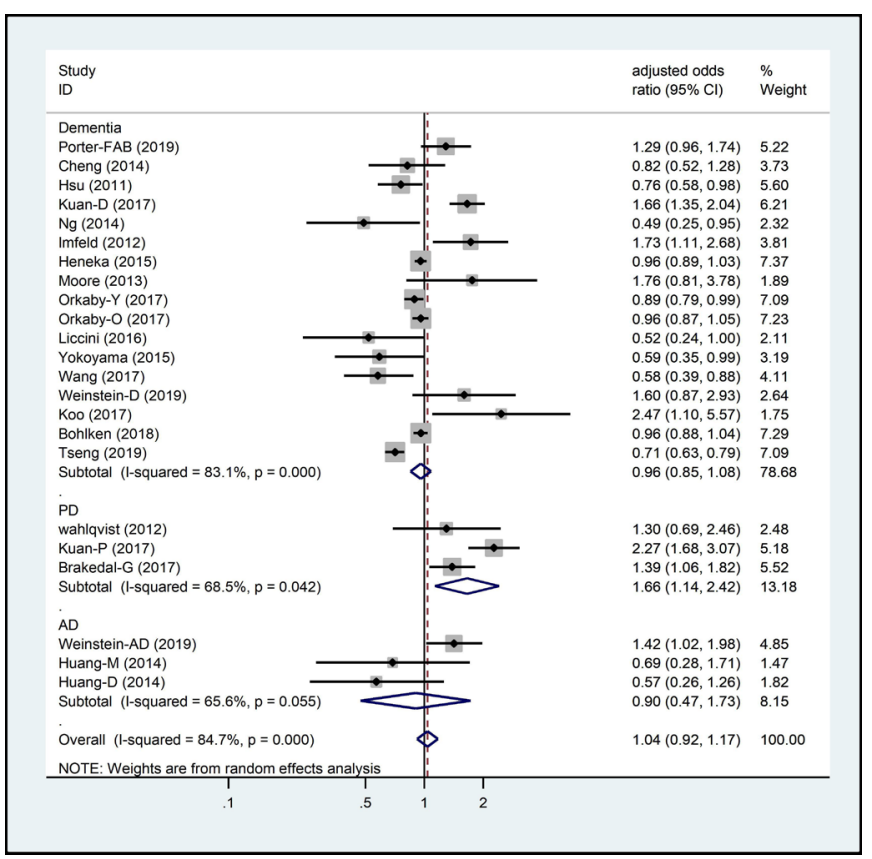

Figure 4 Adjusted OR for metformin exposure on ND incidence subgrouped by subtypes of NDs. ND, neurodegenerative disease. the corresponding pooled ORs were not materially altered for all models. This indicated that no single study influenced the pooled OR qualitatively, suggesting that the outcomes of this meta-analysis was viable.

\section{Metaregression}

In spite of the subgroup analysis performed previously, significant heterogeneity still remained among individual studies. So metaregression was conducted to locate the origins of heterogeneity. As a result, none of the regression coefficients was of statistical significance, revealing that race, study design as well as subtypes of NDs were unable to explain the source of heterogeneity between studies (online supplementary table S7).

\section{DISCUSSION}

As a result of present comprehensive meta-analysis with 285966 participants, we found metformin plays a neutral effect on the risk of NDs in general (pooled adjusted OR $1.04,95 \%$ CI 0.92 to 1.17 ). It was found in subgroup analysis that the role of metformin in the pathogenesis of cognitive impairment with different severity, from mild cognitive impairment determined by different performance scales to dementia, yielded an OR of 0.96 (95\% CI 0.85 to 1.08$)$. As a specific type of dementia, $\mathrm{AD}$ has been studied as a primary outcome with similar combined OR $(0.9,95 \%$ CI 0.47 to 1.73$)$ as other types of cognitive decline.

So far, there are a few interventional human studies to assess metformin effect on cognitive function. A pilot study $(\mathrm{n}=80)$ performed by Luchsinger et al in 2016 found metformin treatment for a year can only marginally improve the selective reminding score compared with the control group in patients with mild cognitive impairment. ${ }^{25}$ Another cross-over RCT study of 20 participants with cognitive impairment due to $\mathrm{AD}$ also showed metformin treatment for 8 weeks improved in a measure of executive function but not in other cognitive score. ${ }^{26}$ However, these RCT studies lacked sufficient exposure time and subjects to evaluate the effect of metformin on ND incidence in patients with diabetes.

The meta-analysis conducted by Fang et al in $2015^{27}$ failed to find a protective effect (RR $0.79,95 \%$ CI 0.82 to $1.01)$, in which only six observational studies evaluating the impact of metformin on dementia were included. A more recent meta-analysis conducted in 2017 by Campbell et al reached a conclusion that metformin can reduce the cognitive decline and dementia incidence in patients with DM. ${ }^{28}$ Since then, several observational studies with large sample sizes have been continuously reported (Kuan et $a l^{7}$ Weinstein et $a l^{9}$ Porter et $a l^{21}$ Bohlken et $a l,{ }^{24}$ Koo et $a l^{10}$ and Tseng. ${ }^{15}$ Among them, only Tseng et al found the protective effect of metformin on cognitive decline. These made it possible to include much more comparisons evaluating the association of metformin with risk of cognitive disorders in the present meta-analysis. This may partly explain that our results are somewhat 
inconsistent with the previous two meta-analysis studies. In addition, our meta-analysis also included studies on PD incidence, another age-related disorder of NDs. This might be another reason for the difference on the overall outcomes.

In contrast, subgroup analysis found metformin exposure increased the risk of PD by $66 \%$ (OR $1.66,95 \%$ CI 1.14 to 2.42). The results should be interpreted with caution for only three cohort studies included, and some confounding factors may interfere with the results. In order to evaluate the effect of metformin solely, only the comparison outcomes of metformin as monotherapy group and non-pharmacotherapy group in the study performed by Wahlqvist $e t$ al were included, while the data of metformin as combined treatment were not included. ${ }^{11}$ The study reported by Brakedal et al, which was based on the Norwegian Prescription Database, compared the incidence of PD between the metformin monotherapy group and the glitazone therapy group with or without metformin. ${ }^{8}$ The results of increased PD risk found in the metformin solo group would inevitably be confounded by the effect of the glitazones. However, it is noteworthy that Kuan et al found metformin exposure increased risk of PD occurrence compared with non-metformin controls in both duration and dosedependent ways. ${ }^{7}$

To the best of our knowledge, whether metformin plays the beneficial or detrimental role in various NDs remains inconclusive, in spite of some compelling evidence that suggests its positive effect against age-related NDs. ${ }^{29}$ Metformin, as one of the first-line pharmacological agents for T2DM treatment, has growing evidence for retarding aging in model organisms and reducing agingrelated diseases. ${ }^{30}$ Metformin can penetrate the bloodbrain barrier; its concentration in cerebrospinal fluid is about one-tenth of that in plasma. ${ }^{26}$ Cellular energy metabolism alterations due to adenosine monophosphate (AMP)-activated protein kinase (AMPK) activation are thought to be the main effect of metformin. So it can be hypothesized that metformin may act on the energy balance of CNS neurons. Metformin was found to decrease phosphor-Ser129 $\alpha$-synuclein, ${ }^{31}$ as well as rescue TH-positive dopaminergic neurons by inhibiting several proinflammatory markers and brain oxidative stress levels via autophagy and mitochondria Reactive Oxygen Species (ROS) clearance. ${ }^{32}{ }^{33}$ Although metformin was reported to exert a neuroprotective role in most of the PD animal models induced by neurotoxins, ${ }^{34}$ it failed to improve motor coordination/balance ${ }^{35}$ and even exacerbated dopaminergic damage in response to 1-methyl-4-ph enyl-1,2,3,6-tetrahydropyridine (MPTP) ${ }^{36}$ which was the most frequently used neurotoxin to evaluate the effect of metformin on models of PD. Another study reported that the overactivation of AMPK might lead to the accumulation of $\alpha$-syn. ${ }^{37}$ All these aforementioned animal studies applied different PD models induced by neuron toxin regimen, which was hard to replicate the idiopathic PD with strong heterogeneity. AMPK activation was found to play a dual role in cognitive decline. It was demonstrated to inhibit the production of $\beta$-amyloid peptide $(\mathrm{A} \beta)$ as well as the phosphorylation of tau. ${ }^{38}{ }^{39}$ However, upregulation of AMPK will increase the production of $A \beta$ through enhancing neuron beta-site amyloid precursor protein (APP)-cleaving enzyme $1 .{ }^{40}$

A reasonable explanation for this paradox is that the effect of metformin on neurodegenerative processes may vary in species, cell types or underlying metabolic state. ${ }^{29}$ In addition, it has been established that longterm metformin intake is associated with the deficiency of vitamin $\mathrm{B}_{12}{ }^{41}$ Recent clinical studies have shown that low vitamin $B_{12}$ levels in serum and cerebrospinal fluid predict greater worsening of PD clinical progression. ${ }^{42} 43$ This mechanism may also involve the detrimental effects of metformin on cognitive function. Moore et al found the association between metformin and cognitive decline was significantly weakened after adjusting for vitamin $\mathrm{B}_{12}$ levels. ${ }^{23}$ As a preventable and treatable side effect, vitamin $\mathrm{B}_{12}$ level monitoring and appropriate supplementation should be strongly recommended, especially in diabetic patients with NDs or high risk of NDs.

There are several limitations of this meta-analysis. First, the considerable heterogeneity was revealed despite the use of random-effects models. Sensitivity analysis was conducted, and not a single study was found as the heterogeneity source. Subgroup analysis based on subtypes of NDs, race, study design and study quality classification was performed. There was no heterogeneity detected within the subgroups except for subtypes of ND; thus, a metaregression was performed. None of the regression coefficients was found to be the significant sources of heterogeneity. The diagnosis discrepancies, uncertainties of metformin exposure and different status of control groups may contribute to an increase of heterogeneity. Second, the sample size of three studies was less than 500 subjects, which may be insufficient to draw a reliable conclusion. ${ }^{162023}$ Third, the adjusted factors in each study were different, and some important confounders, such as HbA1c, duration of diabetes and levels of vitamin $\mathrm{B}_{12}$, were not controlled.

Notwithstanding its limitations, this study does suggest metformin exposure is a potential risk for PD incidence. Therefore, all the future investigations of metformin that carried out for other purposes should include monitoring of neurological function especially the dopaminergic neurons involved.

\section{CONCLUSION}

A total of 23 comparisons out of 19 studies evaluating metformin effect on the onset of both various cognitive decline and PD were included in the present metaanalysis; most of them can be considered as high quality. In general, metformin exposure has a neutral effect on the incidence of general NDs (OR 1.04, 95\% CI 0.92 to 1.17) with considerable heterogeneity. It is well known that long-term use of metformin leads to vitamin $B_{12}$ 
malabsorption, which may aggravate the disease of CNS. Therefore, the subjects with long-term metformin exposure should be monitored for vitamin $B_{12}$ levels and supplemented if necessary.

However, it is noteworthy that metformin exposure increased the risk of PD incidence by $66 \%$. Metformin should be carefully selected as the first-line therapy for patients with DM with PD or deemed at high risk of PD. Further studies are required to confirm these findings and to render a better understanding of the underlying pathogenic mechanism.

Acknowledgements The authors thank Dr Lehao Wu for his advice on the English revision and also Professor Tao Xu for his valuable statistical suggestions.

Contributors FP conceived the meta-analysis. FP and NJ developed the search strategy and provided statistical expertise. FP drafted the manuscript and NJ provided internal peer review. All authors contributed to the development of the inclusion criteria, risk of bias assessment strategy and data extraction criteria. FP and $Y L$ responded to external peer review comments. All authors approved the submission. YL is the guarantor and takes responsibility for the contents of the article.

Funding The work was supported by the China International Medical Foundation (CIMF). The views expressed are those of the authors and not necessarily those of the CIMF. The founders had no role in the study design, data collection, analysis, interpretation or writing of the manuscript.

Competing interests None declared.

Patient consent for publication Not required.

Ethics approval The study was approved by the ethics committee of Peking Union Medical College Hospital (approval number JS-2221).

Provenance and peer review Not commissioned; externally peer reviewed.

Data availability statement All data relevant to the study are included in the article or uploaded as supplementary information.

Open access This is an open access article distributed in accordance with the Creative Commons Attribution Non Commercial (CC BY-NC 4.0) license, which permits others to distribute, remix, adapt, build upon this work non-commercially, and license their derivative works on different terms, provided the original work is properly cited, appropriate credit is given, any changes made indicated, and the use is non-commercial. See: http://creativecommons.org/licenses/by-nc/4.0/.

ORCID iD

Yuxiu Li http://orcid.org/0000-0001-7500-0855

\section{REFERENCES}

1 Honig LS, Vellas B, Woodward M, et al. Trial of solanezumab for mild dementia due to Alzheimer's disease. N Engl J Med 2018;378:321-30.

2 GBD 2016 Dementia Collaborators. Global, regional, and national burden of Alzheimer's disease and other dementias, 1990-2016: a systematic analysis for the global burden of disease study 2016. Lancet Neurol 2019;18:88-106.

3 GBD 2016 Parkinson's Disease Collaborators. Global, regional, and national burden of Parkinson's disease, 1990-2016: a systematic analysis for the global burden of disease study 2016. Lancet Neurol 2018;17:939-53.

4 Kullmann S, Kleinridders A, Small DM, et al. Central nervous pathways of insulin action in the control of metabolism and food intake. Lancet Diabetes Endocrinol 2020;8:524-34.

5 Singh S, Singh PP, Singh AG, et al. Statins are associated with a reduced risk of hepatocellular cancer: a systematic review and metaanalysis. Gastroenterology 2013;144:323-32.

6 Deng G, Yin L, Liu W, et al. Associations of anthropometric adiposity indexes with hypertension risk: a systematic review and metaanalysis including PURE-China. Medicine 2018;97:e13262.

7 Kuan Y-C, Huang K-W, Lin C-L, et al. Effects of metformin exposure on neurodegenerative diseases in elderly patients with type 2 diabetes mellitus. Prog Neuropsychopharmacol Biol Psychiatry 2017;79:77-83.
8 Brakedal B, Flønes I, Reiter SF, et al. Glitazone use associated with reduced risk of Parkinson's disease. Mov Disord 2017;32:1594-9.

9 Weinstein G, Davis-Plourde KL, Conner S, et al. Association of metformin, sulfonylurea and insulin use with brain structure and function and risk of dementia and Alzheimer's disease: Pooled analysis from 5 cohorts. PLoS One 2019;14:e0212293.

10 Koo BK, Kim LK, Lee JY, et al. Increased risk of cognitive impairment by metformin in elderly patients with diabetes in a prospective cohort. Diabetes 2017;66:A399.

11 Wahlqvist ML, Lee M-S, Hsu C-C, et al. Metformin-inclusive sulfonylurea therapy reduces the risk of Parkinson's disease occurring with type 2 diabetes in a Taiwanese population cohort. Parkinsonism Relat Disord 2012;18:753-8.

12 Hsu C-C, Wahlqvist ML, Lee M-S, et al. Incidence of dementia is increased in type 2 diabetes and reduced by the use of sulfonylureas and metformin. J Alzheimers Dis 2011;24:485-93.

13 Cheng C, Lin C-H, Tsai Y-W, et al. Type 2 diabetes and antidiabetic medications in relation to dementia diagnosis. J Gerontol A Biol Sci Med Sci 2014;69:1299-305.

14 Huang C-C, Chung C-M, Leu H-B, et al. Diabetes mellitus and the risk of Alzheimer's disease: a nationwide population-based study. PLoS One 2014;9:e87095.

15 Chin-Hsiao T. Metformin and the risk of dementia in type 2 diabetes patients. Aging Dis 2019;10:37-48.

$16 \mathrm{Ng}$ TP, Feng L, Yap KB, et al. Long-Term metformin usage and cognitive function among older adults with diabetes. $J$ Alzheimers Dis 2014;41:61-8.

17 Heneka MT, Fink A, Doblhammer G. Effect of pioglitazone medication on the incidence of dementia. Ann Neurol 2015;78:284-94.

18 Orkaby AR, Cho K, Cormack J, et al. Metformin vs sulfonylurea use and risk of dementia in US veterans aged $\geq 65$ years with diabetes. Neurology 2017:89:1877-85.

19 Yokoyama H, Ogawa M, Honjo J, et al. Risk factors associated with abnormal cognition in Japanese outpatients with diabetes, hypertension or dyslipidemia. Diabetol Int 2015;6:268-74.

20 Liccini A, Malmstrom TK, Morley JE. Metformin use and cognitive dysfunction among patients with diabetes mellitus. J Am Med Dir Assoc 2016:17:1063-5.

21 Porter KM, Ward M, Hughes CF, et al. Hyperglycemia and metformin use are associated with B vitamin deficiency and cognitive dysfunction in older adults. J Clin Endocrinol Metab 2019;104:4837-47.

22 Imfeld P, Bodmer M, Jick SS, et al. Metformin, other antidiabetic drugs, and risk of Alzheimer's disease: a population-based casecontrol study. J Am Geriatr Soc 2012;60:916-21.

23 Moore EM, Mander AG, Ames D, et al. Increased risk of cognitive impairment in patients with diabetes is associated with metformin. Diabetes Care 2013;36:2981-7.

24 Bohlken J, Jacob L, Kostev K. Association between the use of antihyperglycemic drugs and dementia risk: a case-control study. $J$ Alzheimers Dis 2018:66:725-32.

25 Luchsinger JA, Perez T, Chang H, et al. Metformin in amnestic mild cognitive impairment: results of a pilot randomized placebo controlled clinical trial. J Alzheimers Dis 2016;51:501-14.

26 Koenig AM, Mechanic-Hamilton D, Xie SX, et al. Effects of the insulin sensitizer metformin in Alzheimer disease: pilot data from a randomized placebo-controlled crossover study. Alzheimer Dis Assoc Disord 2017:31:107-13.

27 Ye F, Luo Y-J, Xiao J, et al. Impact of insulin sensitizers on the incidence of dementia: a meta-analysis. Dement Geriatr Cogn Disord 2016:41:251-60.

28 Campbell JM, Stephenson MD, de Courten B, et al. Metformin use associated with reduced risk of dementia in patients with diabetes: a systematic review and meta-analysis. J Alzheimers Dis 2018;65:1225-36.

29 Rotermund C, Machetanz G, Fitzgerald JC. The therapeutic potential of metformin in neurodegenerative diseases. Front Endocrinol 2018;9:400

30 Soukas AA, Hao H, Wu L. Metformin as anti-aging therapy: is it for everyone? Trends Endocrinol Metab 2019;30:745-55.

31 Pérez-Revuelta BI, Hettich MM, Ciociaro A, et al. Metformin lowers Ser-129 phosphorylated $\alpha$-synuclein levels via mTOR-dependent protein phosphatase 2A activation. Cell Death Dis 2014;5:e1209.

32 Lu M, Su C, Qiao C, et al. Metformin prevents dopaminergic neuron death in MPTP/P-Induced mouse model of Parkinson's disease via autophagy and mitochondrial ROS clearance. Int $J$ Neuropsychopharmacol 2016;19:pyw047.

33 Patil SP, Jain PD, Ghumatkar PJ, et al. Neuroprotective effect of metformin in MPTP-induced Parkinson's disease in mice. Neuroscience 2014:277:747-54. 
34 Paudel YN, Angelopoulou E, Piperi C, et al. Emerging neuroprotective effect of metformin in Parkinson's disease: a molecular crosstalk. Pharmacol Res 2020;152:104593.

35 Adedeji HA, Ishola IO, Adeyemi OO. Novel action of metformin in the prevention of haloperidol-induced catalepsy in mice: potential in the treatment of Parkinson's disease? Prog Neuropsychopharmacol Biol Psychiatry 2014;48:245-51.

36 Ismaiel AAK, Espinosa-Oliva AM, Santiago M, et al. Metformin, besides exhibiting strong in vivo anti-inflammatory properties, increases MPTP-induced damage to the nigrostriatal dopaminergic system. Toxicol Appl Pharmacol 2016;298:19-30.

37 Jiang P, Gan M, Ebrahim AS, et al. Adenosine monophosphateactivated protein kinase overactivation leads to accumulation of $\alpha$-synuclein oligomers and decrease of neurites. Neurobiol Aging 2013;34:1504-15.

38 Vingtdeux V, Giliberto L, Zhao H, et al. Amp-Activated protein kinase signaling activation by resveratrol modulates amyloid-beta peptide metabolism. J Biol Chem 2010;285:9100-13.
39 Domise M, Didier S, Marinangeli C, et al. Amp-Activated protein kinase modulates tau phosphorylation and tau pathology in vivo. $\mathrm{Sci}$ Rep 2016;6:26758.

40 Chen Y, Zhou K, Wang R, et al. Antidiabetic drug metformin (GlucophageR) increases biogenesis of Alzheimer's amyloid peptides via up-regulating BACE1 transcription. Proc Natl Acad Sci U S A 2009;106:3907-12.

41 Aroda VR, Edelstein SL, Goldberg RB, et al. Long-Term metformin use and vitamin B12 deficiency in the diabetes prevention program outcomes study. J Clin Endocrinol Metab 2016;101:1754-61.

42 Christine CW, Auinger P, Saleh N, et al. Relationship of cerebrospinal fluid vitamin B12 status markers with Parkinson's disease progression. Mov Disord 2020. doi:10.1002/mds.28073

43 Christine CW, Auinger P, Joslin A, et al. Parkinson study GroupDATATOP Investigators. vitamin B12 and homocysteine levels predict different outcomes in early Parkinson's disease. Mov Disord 2018;33:762-70.

44 Wang C-P, Lorenzo C, Habib SL, et al. Differential effects of metformin on age related comorbidities in older men with type 2 diabetes. J Diabetes Complications 2017;31:679-86. 